of chronic or recurrent abdominal pain were analysed to assess if there were any clinically significant findings, and the cases followed up to ascertain if there was any benefit toward management.

Results 20\% of abdominal ultrasounds were requested for chronic abdominal pain. A total of 132 children had an ultrasound for CAP of which $43 \%$ were male and $56 \%$ were female, with the average age being 8 years. $83 \%$ of scans were requested by GPs. 16\% were requested from paediatric outpatient clinic with half of these patients having sickle cell disease. $1.5 \%$ were requested from the paediatric emergency department.

Only $28 \%$ of all scans requested had alarm symptoms identified. Of those that had alarm symptoms, a third had a significant finding. Of those without alarm symptoms, only 2 had significant findings: splenomegaly and haemorrhagic ovarian cyst. $65 \%$ (86) of all scans were normal. 35\% (46) had positive findings, the most common being mesenteric lymphadenopathy $(29,63 \%) .11$ out of 132 (8.3\%) scans performed identified a diagnostic cause of abdominal pain of which 9 had red flags. Findings included gallstones, splenomegaly, changes in the appendix, hepatosplenomegaly, haemorrhagic ovarian cyst, sclerosing cholangitis and constipation. Incidental findings included renal tract abnormalities and free fluid.

$36 \%(47 / 132)$ of patients were seen by secondary care. $25 \%$ of patients with normal findings and $35 \%$ with mesenteric lymphadenopathy were referred to secondary care services. One patient with a normal ultrasound was subsequently diagnosed with ulcerative colitis and another with sclerosing cholangitis was diagnosed with Crohn's disease. All follow up scans (10) for mesenteric lymphadenopathy showed improvement with no further intervention. 9 patients $(7 \%)$ with CAP were diagnosed with functional abdominal pain syndrome.

Conclusions The majority of ultrasound scans in our DGH were requested by primary care. Alarm symptoms in the history correlated strongly with a clinically significant abnormal finding. The data agrees with current knowledge that the majority of chronic abdominal pain has no discernible pathology and that abdominal ultrasound scans in the absence of alarm symptoms does not yield useful findings. In our population, mesenteric lymphadenopathy was found commonly and did not significantly affect the outcome for our patients but resulted in a significant number of repeat scans and referral to secondary care. Ultrasonography is a useful investigation in paediatric abdominal pain but not in the absence of alarm features. There is scope to improve the utilisation of this resource particularly within primary care.

\section{P56 TWO YEAR FOLLOW UP OF CHILDREN WITH INFLAMMATORY BOWEL DISEASE (IBD) TREATED WITH VEDOLIZUMAB AND USTEKINUMAB}

Roxana Mardare, Natasha Burgess, Dominic Studart, Protima Deb, Marco Gasparetto, NM Croft, Ahmed Kadir, Sandhia Naik. Royal London Hospital

\subsection{6/flgastro-2021-bspghan.65}

Introduction In 2018, our Trust approved the use of Vedolizumab in children with Ulcerative Colitis (UC) and Ustekinumab in children with Crohn's Disease (CD). At the time, access to these drugs for children was only possible through research studies.
Aim Our aim was to assess the efficacy and safety of these novel treatments in our cohort.

Methods We conducted an observational single centre cohort study. Data was obtained from our electronic system, Cerner Millennium, and Infoflex database. Analysis was performed using SPSS.

Results 27 children were treated with Vedolizumab or Ustekinumab with 1 receiving both. All patients had failed anti-TNF medication, except 1 research patient who commenced on Vedolizumab at diagnosis. All patients underwent endoscopy prior to initiating Vedolizumab or Ustekinumab.

Clinical remission was defined as PUCAI $<10$ and $\mathrm{PCDAI}<10$. There was a higher induction rate of remission than quoted in adult studies with similar maintenance of remission. At 2 years follow up, 55\% (15/27) remained in remission, on treatment. $1 / 27$ is currently still on Ustekinumab with mildly active $C D$ and $1 / 27$ had their Vedolizumab stopped due to compliance and monitoring issues. All 10 children currently receiving Vedolizumab remain in remission. 5/6 currently on Ustekinumab remain in remission. Of the $10 / 27$ who failed treatment, 50\% were primary non-responders and $50 \%$ had secondary loss of response. 9/10 required subtotal colectomy and ileostomy, while the research patient, who was anti-TNF naïve, switched to Infliximab after failing Vedolizumab.

There were no serious adverse events apart from one patient who developed eosinophilic pneumonitis, but it is unclear whether this was due to Vedolizumab or 5 ASA. Minor skin or upper respiratory tract infections were diagnosed in 5/10 patients on Ustekinumab and $1 / 10$ patient developed Clostridium difficile. Adrenal insufficiency, as a result of prolonged courses of steroids, was detected in $7 / 27$ children.

Abstract P56 Table 1 Results. *Data expressed as median (range)

\begin{tabular}{|c|c|c|}
\hline & Vedolizumab & Ustekinumab \\
\hline $\mathrm{N}$ & 17 & 10 \\
\hline Median age at diagnosis (years) & $9.1(4.7-14.4)$ & $7.0(4.0-12.4)$ \\
\hline Diagnosis n (M:F) & $\begin{array}{l}\text { UC } 12(6: 6) \\
\text { IBDU } 3(2: 1) \\
\text { CD }(2-\text { research })(1: 1)\end{array}$ & CD $10(5: 5)$ \\
\hline Disease location & E4 14 & L3 8 \\
\hline (Paris classification) $n$ & $\begin{array}{l}\text { E2 } 1 \\
\text { L2L4apG0 } 2\end{array}$ & $\begin{array}{l}\text { L2 } 2 \\
\text { Upper involvement 50\% } \\
\text { Peri anal disease } 70 \% \\
\text { Growth failure } 70 \%\end{array}$ \\
\hline $\begin{array}{l}\text { Length of disease prior to anti-TNF } \\
\text { treatment (months) }\end{array}$ & $4.8(0-44.7)$ & $24.3(6.8-50.3)$ \\
\hline Treatment length (months) & $13.5(2.6-41.2)$ & $22.0(7.1-28.1)$ \\
\hline Follow up length (years) & $4.1(2.0-12.4)$ & $8.0(2.8-11.9)$ \\
\hline Clinical Remission (\%) & Week $1276 \%$ & Week $850 \%$ \\
\hline Note not all patients have received & Week $52 \mathrm{n}=9$ & Week $52 n=4$ \\
\hline treatment for 52 or 104 weeks & Week $104 n=2$ & Week $104 n=3$ \\
\hline
\end{tabular}

Conclusion In children with refractory IBD failing anti-TNF treatment, Vedolizumab and Ustekinumab are effective and safe alternatives for inducing and maintaining remission, avoiding major invasive surgery. 